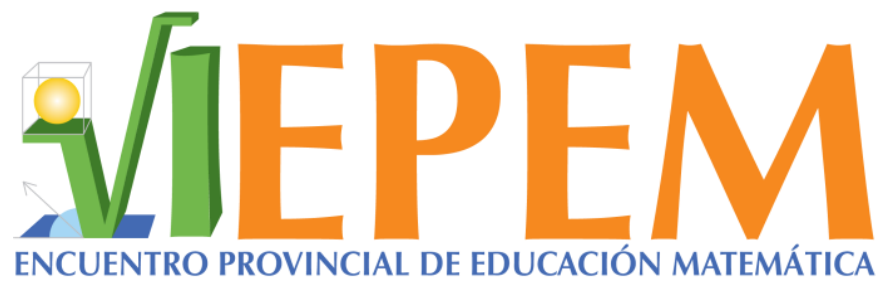

VI Encuentro Provincial de Educación Matemática.

27 al 29 de setiembre, 2017. Puntarenas, Costa Rica.

\title{
Indicadores para valorar la calidad de la enseñanza en Matemática
}

\author{
Jonathan Espinoza González \\ jonaespinoza@una.cr \\ Universidad Nacional \\ Costa Rica \\ Miguel Picado Alfaro \\ miguel.picado.alfaro@una.cr \\ Universidad Nacional \\ Costa Rica
}

\begin{abstract}
Resumen
En la última década se han detectado algunas dificultades en la enseñanza y aprendizaje de las matemáticas en Costa Rica. Así lo evidencian, por ejemplo, los resultados en las pruebas de Bachillerato y el diagnóstico realizado a docentes de Matemática de Secundaria en el año 2010 (Cuarto Informe Estado de la Educación, 2013). En particular, este último pone en realce limitaciones que tienen algunos docentes en cuanto al dominio de ciertos conceptos matemáticos. En este taller se compartirán algunos elementos para analizar la calidad matemática de la instrucción de un profesor cuando enseña conceptos matemáticos. Se espera que los participantes reflexionen sobre su práctica docente y que a su vez obtengan recomendaciones y sugerencias que mejoren los procesos de enseñanza y aprendizaje que realizan.

Palabras clave: Educación; Matemática; calidad matemática de la instrucción; secundaria.
\end{abstract}

\section{Introducción}

La calidad matemática de la instrucción es "un compuesto de varias dimensiones que caracterizan el rigor y la riqueza de las matemáticas de la clase, incluyendo la presencia y ausencia de errores matemáticos, explicación y justificación matemática, representaciones matemáticas, y observaciones relacionadas" (Hill, et al., 2008, p. 431). Su estudio debe abordarse tomando en cuenta aspectos relacionados con el conocimiento del contenido

Taller

\footnotetext{
Espinoza, J. y Picado, M. (2017). Indicadores para valorar la calidad de la enseñanza en Matemática. En Y. Morales-López, M. Picado, R. Gamboa, C. Martínez, M. Castillo y R. Hidalgo (Eds.), Memorias del VI Encuentro Provincial de Educación Matemática, Costa Rica, 2017 (pp. 3840). Heredia: Universidad Nacional. ISBN: 978-9968-9661-5-3. DOI:

http://dx.doi.org/10.15359/epem.6.9
} 
matemático y el conocimiento pedagógico del contenido (Ball, Thames y Phelps, 2008). Los autores de este taller han estudiado estos aspectos enfocando conceptos matemáticos específicos, inscritos en el modelo del Conocimiento Matemático para la Enseñanza propuesto por el grupo de investigación de la Universidad de Michigan (EEUU) (Rodríguez, Picado y Espinoza, 2015; Rodríguez, Picado, Espinoza y Rojas, en prensa; Rodríguez, Picado, Espinoza, Rojas y Flores, 2016; Picado y Espinoza, 2016; Picado y Espinoza en prensa).

El estudio de la calidad matemática de la instrucción que se propone se basa en un sistema de categorías generales y específicas (Hill, et al., 2008). Las categorías generales valoran aspectos como el nivel de profundidad de las matemáticas ofrecidas por el docente, si el docente puede entender y responder adecuadamente las producciones matemáticas de los estudiantes y a los errores e imprecisiones cometidas por el docente durante la instrucción.

Las categorías específicas destacan elementos relacionados con las explicaciones matemáticas, la diversidad de procedimientos, el uso del lenguaje matemático, la atención de errores y dificultades de los estudiantes, las respuestas ofrecidas por el docente, los errores matemáticos, imprecisiones en el lenguaje o notación matemática y la falta de claridad del docente durante la instrucción.

En general, la importancia de valorar la calidad matemática de la instrucción radica no solo en las sugerencias y mejoras que puedan realizarse al proceso de enseñanza analizado, si no que también contribuye a mejorar la práctica docente de quien realiza el análisis desde la reflexión sobre la misma práctica.

\section{Metodología del taller}

El taller se realizará empleando una metodología participativa, también se incentivará el trabajo colaborativo mediante el trabajo en subgrupos. Se espera que los participantes de cada subgrupo brinden aportes significativos a partir de su experiencia como docente y de los conocimientos que adquiera durante el taller.

Para llevar a cabo la valoración de la calidad matemática de la instrucción se observarán y analizarán videos con fragmentos de la enseñanza que realiza un docente de matemática sobre los conceptos básicos de función en cuarto año de la educación secundaria costarricense.

El taller contempla una fase teórica y otra práctica. En la fase teórica se analizará la propuesta de Hill, et al., (2008) sobre la calidad matemática de la instrucción y en la práctica los participantes valorarán la calidad matemática de la instrucción empleando el sistema de categorías y subcategorías analizado en la fase teórica y los fragmentos de video.

Para concluir con el taller, se reflexionará sobre la pertinencia del marco teórico de referencia empleado y sobre cómo este puede brindar sugerencias y recomendaciones que contribuyan a mejorar la enseñanza de conceptos matemáticos en la Educación Secundaria en Costa Rica. 


\section{Resultados esperados}

Se espera que los docentes participantes reflexionen sobre su propia práctica docente, en particular sobre la calidad matemática de la instrucción propia. Que la auto-reflexión provoque la necesidad de conocer un referente teórico para abordar este análisis de una forma sistemática y que el análisis realizado les sugiera recomendaciones y mejoras a la instrucción que realizan en sus clases de Matemática.

\section{Referencias}

Ball, D., Thames, M. y Phelps, G. (2008). "Content Knowledge for Teaching: What Makes It Special?", Journal of Teacher Education, 59(5); 389-407.

Hill, H. C., Blunk, M. L., Charalambous, C. Y., Lewis, J. M., Phelps, G. C., Sleep, L., y Ball, D. L. (2008). "Mathematical Knowledge for Teaching and the Mathematical Quality of Instruction: An Exploratory Study", Cognition and Instruction, 26(4), 430-511.

Picado, M. y Espinoza, J. (en prensa). Las explicaciones matemáticas de un proferor al enseñar los conceptos básicos de función. Capítulo de libro. Benemérita Universidad Autónoma de Puebla, México.

Picado, M. y Espinoza, J. (2016). Informe sobre el análisis del conocimiento pedagógico para la enseñanza. Documento sin publicar.

Programa Estado de la Nación en Desarrollo Humano Sostenible (2013). Cuarto Informe Estado de la Educación (4 ed.). San José, Costa Rica: Editorama.

Rodríguez, A., Picado, M. y Espinoza, J. (2015). Conocimiento en el Horizonte Matemático de un profesor para enseñar funciones en cuarto año de secundaria en Costa Rica. Actas de la Conferencia Interamericana de Educación Matemática. Tuxtla Gutiérrez, México. En $\quad$ http://xiv.ciaemiacme.org/index.php/xiv_ciaem/xiv_ciaem/paper/ view/352/177, consultada el 01Dic-2016, 3:23 pm.

Rodríguez, A., Picado, M., Espinoza, J. y Rojas, N. (en prensa). "El conocimiento especializado del profesor de matemáticas: un estudio de caso sobre la enseñanza de los conceptos básicos de función", UNICIENCIA, 32(1).

Rodríguez, A., Picado, M., Espinoza, J., Rojas, N. y Flores, P. (2016). “Conocimiento común del contenido que manifiesta un profesor al enseñar los conceptos básicos de funciones: un estudio de caso", UNICIENCIA, 30(1), 1-16.

\section{(a) $\odot \Theta \Theta$}

Esta obra está bajo una licencia de Creative Commons Reconocimiento-NoComercialSinObraDerivada 4.0 Internacional. 